\title{
Molecular Genomic Blood Biomarkers for Neuroendocrine Tumors: The Long and Winding Road from Berzelius and Bence Jones to a Neuroendocrine Destination
}

\author{
Kjell Öberg \\ Department of Endocrine Oncology, University Hospital, Uppsala, Sweden
}

\section{Introduction}

Dr. A. Malczewska and her colleagues at the Neuroendocrine Tumor Center of Excellence at the Medical University of Silesia are to be congratulated on their large study of the clinical utility of the NETest across a broad range of neuroendocrine tumors (NETs) including pancreas, small bowel, and bronchopulmonary tumors [1]. Their very positive assessment fully supports the conclusions of more than 40 papers from diverse authors and institutions throughout Europe and North America. In particular, it is consistent with a recent meta-analysis [2] that demonstrated that the NETest was $>95 \%$ accurate and significantly superior to chromogranin A (CgA) in the diagnosis and management of NETs. There seems to be little doubt that the previous era of monoanalyte measurement of secretory products as biomarkers for NET disease has passed.

The purpose of this editorial is to provide a basis for understanding the evolution of biomarker investigation of NET disease - in particular, to assess the advances in mathematical analyses of gene expression and to evaluate the molecular biological relevance of transcriptomic assessment of a NET and how these contribute to clinical

karger@karger.com www.karger.com/nen

Karger"

BOPEN ACCESS
(C) 2020 The Author(s)

Published by S. Karger AG, Basel

This is an Open Access article licensed under the Creative Commons Attribution-NonCommercial-4.0 International License (CC BY-NC) (http://www.karger.com/Services/OpenAccessLicense), applicable to the online version of the article only. Usage and distribution for commercial purposes requires written permission. management. In addition, it seems timely to discuss what the application of machine learning techniques and algorithmic analysis of NET gene cluster analysis to the prediction of tumor behavior may imply for future clinical management.

\section{The Evolution of Cancer Biomarkers}

In 1807, Jöns Jacob Berzelius (1779-1848) was appointed Professor in Chemistry and Pharmacology at the Karolinska Institute where, between 1808 and 1836, he worked with Anna Sundstrom (1785-1871), the first Swedish female chemist. In 1818, he was elected a member of the Royal Swedish Academy of Sciences and over the next 30 years changed the face of scientific chemistry. After his death, he was regarded as the father of modern chemistry - he separated the study of organic from inorganic chemistry. Of particular relevance was his recognition of the power of mathematics and his development of the theory of chemical equilibria and the law of definite proportions. Berzelius predated by almost two centuries the understanding that novel chemical substances (transcriptomes) could be measured (PCR) and mathemati- 


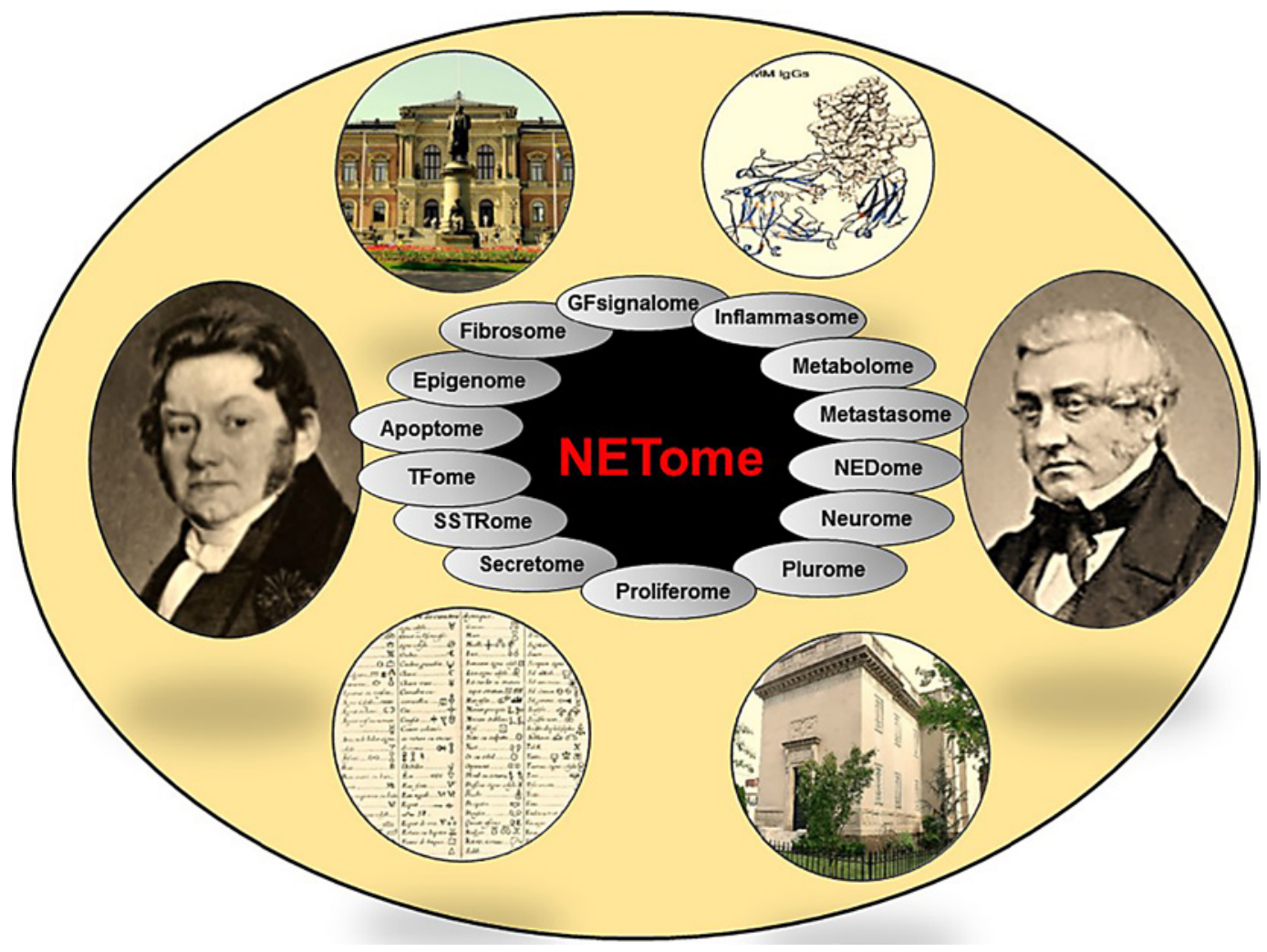

Fig. 1. Jöns Jacob Berzelius (1779-1848) (left) of Uppsala University (top left) was a chemical and mathematical genius who laid the basis for modern chemistry (bottom left) by developing the laws of chemical equilibria, definite proportions, and the periodic table of atomic weights. His memory is enshrined at Yale by the Berzelius Secret Society (bottom right). Henry Bence Jones (1813-1873) (right) at St. George's Hospital London identified albuminosuria (Bence Jones protein) in the urine of a man with "mollities ossium." This compound was subsequently demonstrated to be an im- munoglobulin light chain (top right) diagnostic of the bone marrow neoplasia now referred to as multiple myeloma. Based upon his identification of the re-precipitable protein in the urine of multiple myeloma, Bence Jones is recognized as the progenitor of the science of cancer biomarkers. The omic clusters (center) represent the basis of a multigene blood biomarker for neuroendocrine tumor disease. Permission by Irvin M. Modlin, Yale University School of Medicine, New Haven, CT, USA.

the first cancer biomarker (CB) was a tumor-produced light-chain antibody of immunoglobulin $G$ in multiple myeloma patients, detectable in urine. Subsequently, in 1986, it was identified in serum.

In 1867, Sir Michael Foster (1836-1907) erroneously suggested elevated serum amylase levels (a secretory product) as a biomarker of pancreatic cancer. Thereafter, numerous secretory biomarkers were proposed, including chorionic gonadotropin (choriocarcinoma), catecholamines (pheochromocytoma), and enzymes like acid phosphatase (prostate cancer), alkaline phosphatase (bone), and gastrin in pancreatic NETs [3]. In the late 1970s, carcinoembryonic antigen (colon cancer) and 
CgA for NETs were described. Both have subsequently been negatively evaluated as molecular tools have emerged including mRNAs, circulating tumor DNAs (ctDNAs), circulating tumor cells (CTCs), tumor suppressor genes, oncogenes, nuclear proteins, and telomerase [4].

\section{NET Biomarkers}

Biomarkers are "cellular, biochemical or molecular alterations that are measurable in biological media such as human tissues, cells, or fluids." They comprise agents and technologies that can facilitate the prediction, cause, diagnosis, progression, regression, or outcome of treatment. Such information may be derived from tissue, body fluids, or imaging.

In the NET discipline, cell "secretory products" have been predominantly investigated. These analytes provide little information about tumor molecular biology and are ineffective if the tumor is "nonsecretory." Most are monoanalytes and fail to capture the biological complexity of an evolving tumor. Such information, especially if acquired in real time, is best assessed by multianalyte measurement of mRNA or ctDNA in blood. Tissue assessment is valuable but usually only available at one time point and has significant limitations since random biopsy of a heterogeneous tumor, evolving with time and treatment, has limitations. Alternatively, blood sampling provides safe, repeatable, and real-time information of tumor status. Thus, precise, objective measurements of multiple genes and omic clusters that comprise tumor hallmarks are definable.

Over the course of the last 50 years, a wide variety of agents or their degradation products have been proposed and studied as NET CBs. They include the chromogranins, serotonin, 5-hydroxyindoleacetic acid (5-HIAA), tachykinins, bradykinins, histamine, substance P, insulin, gastrin, and neurokinin A [3]. Suffice it to say that while each has value, their monodimensionality has limited their clinical efficacy.

\section{Blood and Tissue Biomarkers}

Tumor tissue has been the cornerstone of biomarker development and reflects the unescapable fact that a $\mathrm{CB}$ should be unique to the malignant tissue of interest. However, neoplasia reflects a multiplicity of different tissue components and diverse cell systems, including the innate immune response and interactions between neoplastic cells and proximate normal tissue, all evolving with time. Thus, monoanalyte assessments provide limitations in understanding disease development.

Although tissue histopathology should remain a platform, it is subject to the vagaries of descriptive terminology, observer variation, and endless semantic terminology revisions. Ki- 67 has been of value, but its limitations as a monoanalyte are well described. Similarly, its subjective interpretation diminishes this "golden oldie" in an era of molecular genomics and system biology. The concept that a heterogeneous group of complex neoplasia (NETs) can be divided into three categories of "good," "bad," and "ugly" is quixotic and not realistic science in the 21st century [5].

The significant problem of tissue information is heterogeneity and the inability to accurately identify cells clones of disparate lethality. Random sampling is unable to accurately ascertain the course of cell proliferation, invasion, growth factor signaling, and metabolomic activity. Precise, objective identification and measurements are needed to define tumor behavior. Indeed, molecular genomic mathematical analysis of the 14 omic clusters that comprise the NETest score may provide the future basis for multipurpose CB development (Fig. 1). It is important to move beyond the descriptive era (histopathology/immunohistochemistry) of pathology and integrate molecular genomic tools into clinical practice. Since it captures different tumor hallmarks, omic cluster analysis is likely to provide substantial easily accessible biomarker information. This real-time molecular information can be used either as an adjunct to facilitate imaging or as de novo information to define tumor pathobiology. It can also be used to predict therapeutic efficacy (positive predictor quotient [PPQ]), as has already been demonstrated for peptide receptor radionuclide therapy (PRRT).

\section{Biomarker Types and Utility}

The National Institutes of Health (NIH) have proposed three categories of CB. Type 0 markers correlate with diagnosis, prognosis, and outcome, type I markers reflect treatment efficacy, and type II markers are surrogates for clinical endpoints. Individual utility is optimally evaluated in clinical studies structured to conform to biomarker study criteria such as Reporting Recommendations for Tumor Marker Prognostic Studies (REMARK) or the Standards for Reporting of Diagnostic Accuracy (STARD) [6]. Such studies have been lacking in NETs, given the absence of an effective general-purpose multianalyte biomarker (NETest) such as reported by Malczewska et al. [1]. 


\section{The Clinical Relevance of CB Metrics}

It is important to identify the scientific power of a CB. Sensitivity classifies an individual as diseased (positive in disease), while specificity correctly classifies an individual as disease-free (negative in health). Low sensitivity results in a high false-negative rate, while low specificity results in numerous false-positives. Similarly, the assessment of positive predictive value (PPV) and negative predictive value (NPV) defines accuracy. Diagnostic power is best assessed using the area under the curve (AUC); AUCs should be $>0.8$ (moderately accurate). Ideally, a biomarker should exceed $90 \%$ for specificity and $80 \%$ for sensitivity, NPV, or PPV and have an AUC $>0.8$ [4]. In respect of the NETest, numerous studies and a meta-analysis demonstrate values $>85 \%$ and an AUC $>0.9$ [2] compared to $\mathrm{CgA}$, which ranges from 30 to $70 \%$.

\section{Limitations of Current NET Biomarkers}

Current NET biomarkers are monoanalyte assessments of secretory proteins or amines and provide limited information about tumor behavior. A few are effective for diagnosis - e.g., insulin/insulinoma, glucagon/ glucagonoma, and gastrin/gastrinoma - but these individually comprise $<2 \%$ of all tumors. Serotonin and CgA are general markers, but each exhibits limitations.

Serotonin and its degradation product, 5-HIAA, were formerly widely assessed in urine and plasma [4]. 5-HIAA has a high diagnostic specificity $(\sim 100 \%)$ but low sensitivity ( 35\%) [7], minimal prognostic value, and limited correlation with disease severity. Blood serotonin is difficult to measure and urine 5-HIAA cumbersome to assess, and levels are influenced by diverse foods and drugs. These analytes have limited utility since they are only effective in serotonin-secreting lesions ( $\sim 15-20 \%$ of all NETs).

$\mathrm{CgA}$ is a product of the neuroendocrine exocytotic process and exists in the blood as a heterogeneous antigen composition. Some report it to correlate with tumor size and to provide prognostic information, but this is controversial [4]. Measurement is problematic due to different assay incompatibilities and nonspecificity (10-35\%) since numerous diseases, renal failure, and proton pump inhibitors elevate CgA. Meta-analyses of CgA in gastroenteropancreatic NETs indicate a diagnostic sensitivity of $73 \%$ with a $95 \%$ specificity [8]. In bronchopulmonary NETs, this was 35 and 94\% [9]. The suboptimal metrics (sensitivity $>80 \%$ and specificity $>90 \%$ ) have led to diminished enthusiasm for CgA as a NET CB [4].
Pancreastatin, a CgA derivative, may be elevated in $58-81 \%$ of NETs $[4,10,11]$. Its clinical utility is unclear as levels do not correlate with location, and functionality and relationships with tumor aggressiveness or survival are controversial. Despite the fact that it was initially reported as "The long sought for universal, sensitive, specific tumor marker for neuroendocrine tumors," it has not attained clinical acceptance and is now seldom used as a biomarker [12].

\section{Monoanalyte versus Multianalyte Pros/Cons}

A monoanalyte cannot define the cellular activity of a tumor since it does not encompass the numerous biological processes that define neoplasia and its progression (growth factor signaling, metabolic status, cell cycling, etc.) [4]. In NETs, single analytes produced by a specific cell type (EC/ECL/PP/D, etc.) are unlikely to be representative of a heterogeneous group of tumors. Studies in other neoplasias confirm that monoanalyte CBs cannot define the multiple biological processes that comprise cancer. On the contrary, multianalyte analyses capture the transcriptomic drivers (hallmarks of cancer) proposed by Hanahan and Weinberg [13] and provide an assessment of the tumor molecular genomic mechanisms. Tumor biology "regulators," if detectable in blood, e.g., as a liquid biopsy, provide a more accurate reflection of the pathobiology of the tumor as it evolves. The magnitude and complexity of measurements necessary to quantify the multiple regulators of neoplastic cell biology require sophisticated biomathematical analysis. This process generates a numeric score and an algorithmic probability based on tumor transcriptome analysis that can facilitate clinical management. Such techniques have been successfully employed in breast, bronchopulmonary, and blood malignancies [14-16]. They direct therapy and are included in various guidelines as standard of care, e.g., the American Society of Clinical Oncology (ASCO) and the National Comprehensive Cancer Network (NCCN). The information provided by Malczewska et al. [1] demonstrates that this strategy is now applicable to NET disease. More recent sophisticated investigation of NETest "omic cluster analysis" strategies indicates that further mathematical extrapolation may provide additional clinically relevant information to predict tumor behavior [17].

The criteria for an effective CB include safe and easy access to the compartment (blood), availability of realtime information, objective identification of multiple 
(rather than single) components of tumor biology, use of state-of-the-art methodology, and that data interpretation uses artificial intelligence, machine learning, and/or computation to objectivize information. Real-time assessment is a critical factor in clinical management and is ideally provided by sampling the blood compartment using techniques that quantify tumor biology-derived material, e.g., DNA or mRNA. Such "liquid biopsies" represent an innovative tool in precision oncology that overcomes many of the current limitations of monoanalytes and tissue biopsies.

\section{Liquid Biopsy - The New Horizon}

Multianalyte biomarkers in blood include tumor-derived components such as ctDNA, CTCs, RNA (lncRNA, miRNA), extracellular vesicles, and "tumor-educated" platelets. A few have been investigated in NETs.

ctDNA studies have yielded limited clinically actionable information since mutations are not a prominent feature of NETs [18]. Although CTC measurement is attractive, NET studies have been disappointing. Detectable CTCs are evident in $<50 \%$ of patients and cell counting has limitations (monoanalyte). Thus, only partial relationships to therapeutic response and prognosis are possible. Assessment of posttranscriptional regulators in neoplasia (miRNA) to date has inadequate clinical application to NETs. The technology is difficult, with lack of standardization and no consistently detectable blood signature. Some reports have identified "specific" NET miRNAs, but the science remains mostly descriptive and evolving [19].

Since there is little information to support the clinical utility of these approaches in NETs, attention has been directed to the mRNA-based techniques. The current study by Malczewska et al. [1] provides a very convincing assessment of the clinical utility of mRNA measurement. It also provides compelling information demonstrating that a monoanalyte assessment $(\mathrm{CgA})$ is significantly inferior to a multianalyte assessment (NETest).

\section{NETest}

The NETest represents the first successful attempt to provide a multianalyte signature in blood that has clinical utility in the management of NET disease. It measures multiple NET-related transcripts (51) in blood which, when algorithmically analyzed, constitute a tumor "bio- logical signature" that correlates with tissue expression [20]. The signature was derived by transcriptomic evaluation using gene discovery technology in gastrointestinal, pancreatic, and bronchopulmonary tumors followed by evaluation and validation of the assay in training and test sets [21]. An independent NIH study using the gene expression profiles of 10,244 tumors encompassing 32 different types of neoplasia - including NETs and normal samples from the TCGA database - found that the NETest gene signature accurately captured the NET genotype [22]. The same study demonstrated that the gene expression profile was not readily detectable in hematologic-derived cells, particularly "white blood cells," which constitute the predominant source of blood-derived mRNA.

Sample collection is done in a tube with mRNA stabilization buffer which allows storage at room temperature for a week and at $-80^{\circ} \mathrm{C}$ indefinitely. The assay comprises mRNA isolation from whole blood with subsequent cDNA production and qPCR. Specific target analyte measurement demonstrates high reproducibility with a coefficient of variation $<5 \%$ and inter- and intra-assay metrics ranging from 0.4 to $4.8 \%$. The assay has been effectively calibrated and functions independently in two different laboratories (Sarah Cannon Molecular Diagnostics, HCA, London, UK, and Wren Laboratories, Branford, CT, USA) [23].

Results are expressed as an activity index (NETest score) from 0 to 100 . The assay currently uses a cutoff of 20 (upper limit of normal). Values of $21-40 \%$ are considered representative of "stable" disease, while values of $41-100 \%$ reflect "progressive" disease. It is likely that mathematical adjustments of these cutoffs will occur with further usage and as machine learning criteria are applied. Nevertheless, the current metrics far exceed any other NET biomarker. As a diagnostic, it exhibits a sensitivity/specificity of $90-97 \%$. AUCs range from 0.90 to 0.98 versus, e.g., CgA (AUC: 0.64), while PPV and NPV are $>85 \%$. Unlike $\mathrm{CgA}$, there is no alteration associated with food, acid suppressive medication, sex, ethnicity, or age.

\section{Clinical Utility}

Several independent studies provide a positive evaluation of the NETest as a diagnostic and monitor of treatment efficacy in both gastroenteropancreatic and bronchopulmonary NETs. All have determined that accuracy and metrics exceed those of CgA and meet the NIH spec- 
ifications for an effective biomarker. A particularly important use is after surgical tumor resection, where the NETest detects residual disease and predicts early recurrence even in R0 resections. Similarly, its use to differentiate stable from progressive disease is $85-95 \%$ congruent with imaging and can be detected up to 1 year earlier. Apart from diagnostic and disease status evaluation, the NETest can also function as a prognostic. A recent NIH registry study demonstrated that a low NETest $(<40 \%)$ had an excellent outcome (progression-free survival [PFS] not reached), while intermediate and high NETest scores (41-100\%) were associated with significantly shorter PFS and treatment failures [24]. A recent NETest meta-analysis identified the diagnostic accuracy of the NETest to be $95-96 \%$ with a mean diagnostic odds ratio of $>5,000$, a positive likelihood ratio of $\sim 200$, and a negative likelihood ratio of $<0.1$ [2]. As a type 0 marker, the accuracy was $91.5-97.8 \%$. As an interventional/response biomarker (type I), the accuracy was $93.7-97.4 \%$. The NETest was $84.5-85.5 \%$ accurate in differentiating stable from progressive disease (type II marker). The pooled AUC was $0.954 \pm 0.005$. These results further confirm the NETest as an accurate biomarker suitable for clinical use in NET disease management and are consistent with observations regarding the utility of liquid biopsies in other oncological disciplines. A recent review of the NETest [25] raised some concerns about the relationship between the score and both grade and metastatic spread. The paper by Malczewska et al. [1] investigated both these questions, and the results confirmed that the NETest is related to grade and that there is a relationship with metastatic disease. These observations raise the possibility that in the future the use of advanced omic analysis, real-time grading, and staging may be undertaken using a real-time liquid biopsy rather than a "one-time" blind biopsy of heterogeneous tumors. While neither grading nor staging alone are effective as therapy predictors, the need therefore to accurately identify whether a therapy is effective and that disease is progressive is a critical requirement for optimizing management. The paper by Malczewska et al. [1] from an ENETs Centre of Excellence supports reports from many different institutions and confirms the clinical utility of a molecular genomic liquid biopsy for NETs. It provides reproducible, accurate, and clinically reliable information for the physician.

The NETest has been used to monitor the treatment efficacy for PRRT. NETest levels accurately (>95\%) correlate with image-based response assessments, demonstrating that the NETest is an effective surrogate marker (type II) for PRRT radiological response. Early decreases (by cycle II of therapy) were predictive (>85\%) of final response, demonstrating utility as a type I (intervention$\mathrm{al} /$ response) biomarker. In an independent series, the PRRT predictive quotient (PPQ) was 95\% accurate in the prediction of PRRT efficacy [26]. Those predicted as "nonresponders" had a median PFS of 8-14 months compared to a median PFS not reached at 31 months in those predicted as responders. NETest levels and PPQ assessment of PRRT demonstrated a $>90 \%$ efficacy for prediction and treatment monitoring [27]. It seems likely that integration of imaging with objectively derived molecular genomic data will provide a further strategy to amplify the value of the NETest, omic cluster analysis, and a precision-based approach to PRRT, rather than an archaic one-size-fits-all strategy. One hopes that the same "slow adopter" ("I do not understand it") strategy that hindered the adoption of PRRT will not inhibit the application of molecular genomic assessment to facilitating the efficacy of PRRT as a precision medicine therapeutic tool.

\section{Health Economics}

A recent evaluation of US healthcare costs identified that the NETest would decrease diagnostic expenditure by USD 240 to USD 1.69 billion annually. Similarly, the costs of follow-up would have a further additional annual reduction of USD 205 to USD 1.1 billion by decreasing imaging [2]. One study identified that imaging could be reduced by $50 \%$ if stable molecular disease was evident on blood testing [24]. It is likely that the NETest can serve as a noninvasive, easily repeatable investigation which provides a real-time guide to individual disease status and facilitates implementation of optimal care.

\section{Conclusion}

The data presented by Malczewska et al. [1] in this very large series not only substantiate the clinical validity of the NETest, but once again demonstrate the limitations of CgA. Our societies and guidelines should move beyond the measurement of simple one-dimensional secretory markers and accept the introduction of novel technology utilizing real-time mathematical analysis of transcriptome-based disease assessment. We need to advance the science of NET disease manage- 
ment by doing more than just promoting expensive agents of limited efficacy. Only by using the test, understanding its uses and limitations, and gaining experience and knowledge can we progress the discipline into understanding the value of a molecular genomic liquid biopsy in the management of our patients. The test can be ordered electronically anywhere in Europe, the UK, and North America and the data are delivered electronically within 3-5 days. I, who many years ago helped initiate the usage of $\mathrm{CgA}$, am more than satisfied to see it so effectively surpassed by molecular genomic measurement.

New days bring new scientific advances and we need to be receptive...

\section{References}

1 Malczewska A, Witkowska M, Wójcik-Giertuga M, Kuśnierz K, Bocian A, Walter A, et al. Prospective evaluation of the NETest as a liquid biopsy for gastroenteropancreatic and bronchopulmonary neuroendocrine tumours: an ENETS Centre of Excellence experience. Neuroendocrinology. 2020 doi: 10.1159/000508106 [Epub ahead of print].

2 Oberg K, Califano A, Strosberg JR, Ma S, Pape $\mathrm{U}$, Bodei $\mathrm{L}$, et al. A meta-analysis of the accuracy of a neuroendocrine tumor mRNA genomic biomarker (NETest) in blood. Ann Oncol. 2020 Feb;31(2):202-12.

3 Öberg K. The Genesis of the Neuroendocrine Tumors Concept: from Oberndorfer to 2018. Endocrinol Metab Clin North Am. 2018 Sep; 47(3):711-31.

4 Oberg K, Modlin IM, De Herder W, Pavel M, Klimstra D, Frilling A, et al. Consensus on biomarkers for neuroendocrine tumour disease. Lancet Oncol. 2015 Sep;16(9):e435-46.

5 Nildom N, Grebo K. Observationes de Inebriati - Cancer Biomarkers and the Bioinformatic Era. London: Athenaeum; 2020. p. 12 23.

6 Bossuyt PM, Reitsma JB, Bruns DE, Gatsonis CA, Glasziou PP, Irwig LM, et al.; Standards for Reporting of Diagnostic Accuracy. The STARD statement for reporting studies of diagnostic accuracy: explanation and elaboration. Ann Intern Med. 2003 Jan;138(1):W112.

7 Bajetta E, Ferrari L, Martinetti A, Celio L, Procopio G, Artale S, et al. Chromogranin A, neuron specific enolase, carcinoembryonic antigen, and hydroxyindole acetic acid evaluation in patients with neuroendocrine tumors. Cancer. 1999 Sep;86(5):858-65.

8 Yang X, Yang Y, Li Z, Cheng C, Yang T, Wang $\mathrm{C}$, et al. Diagnostic value of circulating chromogranin A for neuroendocrine tumors: a systematic review and meta-analysis. PLoS One. 2015 Apr;10(4):e0124884.
9 Malczewska A, Kidd M, Matar S, Kos-Kudła B, Bodei L, Oberg K, et al. An assessment of circulating chromogranin A as a biomarker of bronchopulmonary neuroendocrine neoplasia: a systematic review and meta-analysis. Neuroendocrinology. 2020;110(3-4): 198216.

10 Stridsberg M, Oberg K, Li Q, Engström U, Lundqvist G. Measurements of chromogranin A, chromogranin B (secretogranin I), chromogranin C (secretogranin II) and pancreastatin in plasma and urine from patients with carcinoid tumours and endocrine pancreatic tumours. J Endocrinol. 1995 Jan; 144(1):49-59.

11 Calhoun K, Toth-Fejel S, Cheek J, Pommier R. Serum peptide profiles in patients with carcinoid tumors. Am J Surg. 2003 Jul;186(1): 28-31.

12 Ito T, Igarashi $\mathrm{H}$, Jensen RT. Serum pancreastatin: the long sought universal, sensitive, specific tumor marker for neuroendocrine tumors? Pancreas. 2012 May;41(4):505-7.

13 Hanahan D, Weinberg RA. Hallmarks of cancer: the next generation. Cell. 2011 Mar; 144(5):646-74.

14 Jaeger U, Kainz B. Monitoring minimal residual disease in AML: the right time for real time. Ann Hematol. 2003 Mar;82(3):139-47.

15 Miller WR, Larionov A, Renshaw L, Anderson TJ, Walker JR, Krause A, et al. Gene expression profiles differentiating between breast cancers clinically responsive or resistant to letrozole. J Clin Oncol. 2009 Mar; 27(9):1382-7.

16 Urgard E, Vooder T, Võsa U, Välk K, Liu M, Luo C, et al. Metagenes associated with survival in non-small cell lung cancer. Cancer Inform. 2011;10:175-83.

17 Kidd M, Kitz A, Drozdov IA, Modlin IM. Neuroendocrine tumor omic gene cluster analysis amplifies the prognostic accuracy of the NETest. Neuroendocrinology. 2020 doi: $10.1159 / 000508573$ [Epub ahead of print].

18 Kidd M, Modlin I, Oberg K. Towards a new classification of gastroenteropancreatic neuroendocrine neoplasms. Nat Rev Clin Oncol. 2016 Nov;13(11):691-705.
19 Malczewska A, Kidd M, Matar S, Kos-Kudla B, Modlin IM. A Comprehensive Assessment of the Role of miRNAs as Biomarkers in Gastroenteropancreatic Neuroendocrine $\mathrm{Tu}$ mors. Neuroendocrinology. 2018;107(1):7390.

20 Modlin IM, Kidd M, Malczewska A, Drozdov I, Bodei L, Matar S, et al. The NETest: The Clinical Utility of Multigene Blood Analysis in the Diagnosis and Management of Neuroendocrine Tumors. Endocrinol Metab Clin North Am. 2018 Sep;47(3):485-504

21 Modlin IM, Drozdov I, Kidd M. The identification of gut neuroendocrine tumor disease by multiple synchronous transcript analysis in blood. PLoS One. 2013 May;8(5):e63364.

22 Chen F, Zhang Y, Gibbons DL, Deneen B, Kwiatkowski DJ, Ittmann M, et al. Pan-Cancer Molecular Classes Transcending Tumor Lineage Across 32 Cancer Types, Multiple Data Platforms, and over 10,000 Cases. Clin Cancer Res. 2018 May;24(9):2182-93.

23 Kidd M, Drozdov IA, Matar S, Gurunlian N, Ferranti NJ, Malczewska A, et al. Utility of a ready-to-use PCR system for neuroendocrine tumor diagnosis. PLoS One. 2019 Jun; 14(6):e0218592.

24 Liu E, Paulson S, Gulati A, Freudman J, Grosh W, Kafer S, et al. Assessment of NETest clinical utility in a U.S. registry-based study. Oncologist. 2019 Jun;24(6):783-90.

25 Rindi G, Wiedenmann B. Neuroendocrine neoplasia goes molecular - time for a change. Nat Rev Clin Oncol. 2019 Mar;16(3):149-50.

26 Bodei L, Kidd MS, Singh A, van der Zwan WA, Severi S, Drozdov IA, et al. PRRT genomic signature in blood for prediction of 177Lu-octreotate efficacy. Eur J Nucl Med Mol Imaging. 2018 Jul;45(7):1155-69.

27 Bodei L, Kidd M, Modlin IM, Severi S, Drozdov I, Nicolini S, et al. Measurement of circulating transcripts and gene cluster analysis predicts and defines therapeutic efficacy of peptide receptor radionuclide therapy (PRRT) in neuroendocrine tumors. Eur J Nucl Med Mol Imaging. 2016 May;43(5): 839-51. 